\title{
Quantitative synteny scoring improves homology inference and partitioning of gene families
}

\author{
Raja Hashim Ali, ${ }^{1,2}$, Sayyed Auwn Muhammad ${ }^{1,2,3}$, Mehmood Alam Khan ${ }^{1,2}$, Lars Arvestad ${ }^{2,3,4^{*}}$ \\ From Eleventh Annual Research in Computational Molecular Biology (RECOMB) Satellite Workshop on \\ Comparative Genomics \\ Lyon, France. 17-19 October 2013
}

\begin{abstract}
Background: Clustering sequences into families has long been an important step in characterization of genes and proteins. There are many algorithms developed for this purpose, most of which are based on either direct similarity between gene pairs or some sort of network structure, where weights on edges of constructed graphs are based on similarity. However, conserved synteny is an important signal that can help distinguish homology and it has not been utilized to its fullest potential.

Results: Here, we present GenFamClust, a pipeline that combines the network properties of sequence similarity and synteny to assess homology relationship and merge known homologs into groups of gene families. GenFamClust identifies homologs in a more informed and accurate manner as compared to similarity based approaches. We tested our method against the Neighborhood Correlation method on two diverse datasets consisting of fully sequenced genomes of eukaryotes and synthetic data.

Conclusions: The results obtained from both datasets confirm that synteny helps determine homology and GenFamClust improves on Neighborhood Correlation method. The accuracy as well as the definition of synteny scores is the most valuable contribution of GenFamClust.
\end{abstract}

\section{Background}

Gene family classification is an important pre-requisite in Bioinformatics studies and enables, e.g., phylogenetic and structural analysis. Proteins translated from related genes (homologs) tend to have similar structure and function and most of their chemical properties are also similar [1]. One of the initial tasks in genome analysis, given a novel genome, is to find homology between genes and then to use this homology information to make a rough guess about the properties of each gene as well as to construct the phylogenetic tree from these gene families. Due to the importance of gene family classification, it has become one of the most active fields of research in Bioinformatics and bioinformaticians have employed different algorithms to detect homology and to partition detected homologs into gene families.

\footnotetext{
* Correspondence: arve@nada.su.se

${ }^{2}$ Science for Life Laboratory, Karolinska Institutet Science Park, Solna, Sweden Full list of author information is available at the end of the article
}

The pioneers of homology inference algorithms use similarity-based methods, typically employing BLAST $[2,3]$ as a subroutine, like Reciprocal Bidirectional Hits (RBH) [4] and Clusters of Orthologous Groups (COGs) [5]. Other examples of similar algorithms are SiLiX [6] and BlastClust [7] that apply threshold on BLAST output, e.g., E-value and/or percentage identity, and perform single linkage clustering [8]. Despite speed and simplistic computations, they lack the sensitivity to infer homology for more divergent and highly evolving gene families, e.g., in the presence of differential gene loss and/or domain recombination events [9-11]. The next class of algorithms use sequence clustering techniques and examines a wide range of BLAST hits. Well-known examples are TribeMCL [12], OrthoMCL [13], InParanoid [14], and MultiParanoid [15], which are applicable on large datasets and are more accurate than simple BLAST based methods. The next generation of homology inference algorithms improved the accuracy and the
C Biomed Central

() 2013 Ali et al.; licensee BioMed Central Ltd. This is an open access article distributed under the terms of the Creative Commons Attribution License (http://creativecommons.org/licenses/by/2.0), which permits unrestricted use, distribution, and reproduction in any medium, provided the original work is properly cited. 
time and/or memory complexity requirements and include algorithms like Neighborhood Correlation [16], HiFiX [17], PHYRN [18], COCO-CL [19] etc. and infer homologs by extracting evidence from network structure of BLAST hits or multiple sequence alignments.

The algorithms mentioned previously are all based on sequence similarity. Other algorithms have been designed that do not infer homology between genes but instead retrieve chromosomal regions that share homology. Given the chromosomal homology information, one can infer homologous genes by using similarity matches in the region. Examples are R-window [20] and max-gap [21], which use the concept of "gene teams" (conserved gene clusters) [22]. Popular software that implement these algorithms or variants thereof are SynBlast [23], MCScanX [24], Cyntenator [25] and DAGChainer [26]. However, homology inference from these software require further processing of results and homology is not a direct result from these algorithms and software.

At present, there is a relative lack of methods that assess homology by using synteny heuristics directly and not through implicit computation of syntenic regions. The few algorithms that use synteny directly for homology inference are not able to give an objective quantitative measure of synteny (capture synteny information in a score) for a given pair of gene. As an example, SYNERGY, a species-tree aware and synteny-based method, showed impressive results on yeast dataset [27]. However, the method is not general enough for use with all datasets [28]. An issue for using synteny information in this way is the fragmentation in genome assemblies, which may handicap current synteny based software. Alternative synteny-based strategies that may avoid this pitfall define synteny by using a fixed sized neighborhood (termed local synteny). Jun et al. [29] have used this definition to identify orthologs and have shown comparative results with other similarity-only based approaches. Another approach based on local synteny that also takes into account evidence from multiple genomes is SYNS (SYNtenic teamS) and has been shown to work on five Protoploid yeasts [30]. These and other such strategies generally define homology in the neighborhood by applying a threshold on the BLAST E-values, which has been shown by Joseph et al. [31] to be a weak indicator of homology.

We propose a novel gene similarity and synteny based pipeline that makes use of network structure for both similarity and synteny. First, it is a method based on evidence for conserved gene order across many genomes instead of only two genomes directly. Second, it is the first method to calculate synteny scores based on the Neighborhood Correlation score [31] (NC) instead of BLAST E-value and defines a quantitative synteny score. Third, there is a noticeable gain in accuracy when combining $\mathrm{NC}$ and synteny score compared to $\mathrm{NC}$ alone.
Fourth, the pipeline is robust to fragmentation in genome assemblies and can reliably be employed to most data sets. GenFamClust is available as a single, user-friendly Java command line tool that provides homology inference pipeline and clustering algorithm implementations.

\section{Methods}

Given a full list of sequences in Fasta format and information about order of each gene in a specified format, GenFamClust partitions the data into homologs and non-homologs by determining combined evaluated scores from NC $[16,31]$ and synteny correlation $(\mathrm{SyC})$ scores. From these classified homologs, GenFamClust constructs the gene families by using Single [8], Average [32] or Complete Linkage [33] clustering. GenFamClust searches for evidence of conserved synteny by computing the synteny correlation score for each pair of sequences that have acceptable sequence similarity. The main idea is that the advantages that NC has over BLAST based scores, can also be employed for synteny to make it more robust, standardized and accurate than the "gene teams" concept by making it based on evidence from multiple witnesses. While NC scores over 0.5 can in general be classified as homologs, GenFamClust uses synteny to assess homology for gene pairs with $\mathrm{NC}$ scores below 0.5 .

\section{The data and pipeline}

GenFamClust assumes that there are two sets of data; the query dataset $Q$ and the reference dataset $R$. The query dataset $Q$ consists of those genes for which homology relationships are inquired and classification into gene families is desired. The reference dataset $R$ consists of those genes which will be used for finding evidence for conserved synteny but may not be of interest in the final analysis.

The input expected by the GenFamClust implementation is synteny files that contain information about the gene order and Fasta files containing protein sequences (exactly one per gene). Figure 1 describes the general workflow of the pipeline.

\section{Neighborhood Correlation calculation}

We chose the Neighborhood Correlation score as given by Song et al. [16] as our measure of similarity. The attractive feature about this measure is that it is standardized, has a known range between 0 and 1 , can easily be applied a threshold, has been shown to work well with diverse protein domain architectures and is more accurate than any simple BLAST based thresholds. We demand that NC score is above a threshold $\beta$ and setting $\beta=0.3$ ensures that most non-homologs are discarded while retaining virtually all homologs in the dataset. Furthermore, this limit helps reduce memory consumption. NC needs a lenient 


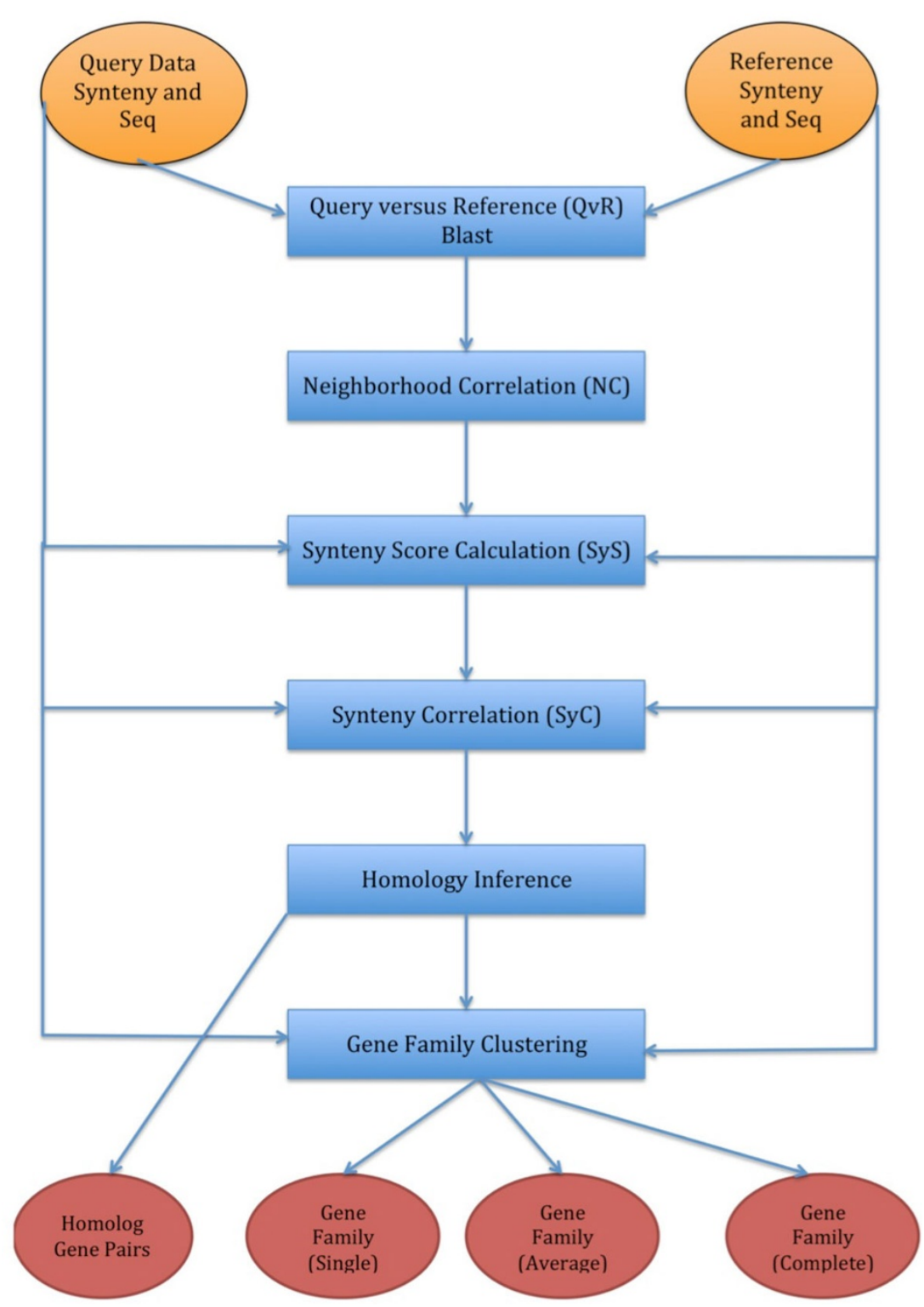

Figure 1 General workflow of the GenFamClust pipeline. Orange circles: the input to the pipeline; blue squares: module or process of the pipeline; red circles: the output of the pipeline. Arrows indicate data flow of the pipeline.

threshold on BLAST E-value [16]; For our experiments, we have chosen $\mathrm{E}=0.1$.

\section{Synteny score calculation}

To compute SyC, we make use of a synteny score SyS $\left(g_{1}, g_{2}\right)$ for two sequences $g_{1}$ and $g_{2}$. Let $\mathrm{n}(g)$ be the set of neighbor genes, upstream or downstream of $g$, at most at distance $k$, on a chromosome or contig. We define $\operatorname{SyS}\left(g_{1}, g_{2}\right)=\max \left\{\mathrm{NC}(a, b): a \in \mathrm{n}\left(g_{1}\right), b \in \mathrm{n}\left(g_{2}\right)\right\}$.

The purpose of $S y S$ is to find evidence of homology of genes in $\mathrm{n}\left(g_{1}\right)$ with genes in $\mathrm{n}\left(g_{2}\right)$. SyS is only calculated for pairs $\left(g_{1}, g_{2}\right)$ where $\mathrm{NC}\left(g_{1}, g_{2}\right)>\beta$ and at least one of $g_{1}$ and $g_{2}$ is in Q. Below $\beta, \mathrm{NC}$ is regarded sufficient to indicate that no homology exists. While the $Q x Q$ gene pairs indicate direct evidence for synteny in the query dataset, the $Q x R$ gene pairs provide indirect evidence within the reference dataset genes. Our experiments with the human-mouse dataset suggests setting $k=5$ (see Additional File 1).

We tried four different functions to define a synteny score for a pair of genes and an assessment of the behavior of each method made us choose the "Maximum Score" method. See Additional File 1 for details on the alternatives and the assessment. 


\section{Syntenic correlation calculation}

For each gene pair $\left(g_{1}, g_{2}\right)$ such that $g_{1}, g_{2} \in Q$ and NC $\left(g_{1}, g_{2}\right)>\beta$, GenFamClust computes synteny correlation scores, SyC, for using pairs with good NC score. Let $H=n c H i t s\left(g_{i}\right) \cap n c H i t s\left(g_{j}\right)$ and

$H=n c H i t s\left(g_{i}\right) \cap n c H i t s\left(g_{j}\right)$, then

$$
\operatorname{SyC}\left(g_{i}, g_{j}\right)=\frac{\sum_{h \in H}\left(S \gamma S\left(g_{i}, h\right)-\overline{S y S}\left(g_{i}\right)\right)\left(S y S\left(g_{j}, h\right)-\overline{S \gamma S}\left(g_{j}\right)\right)}{\sqrt{\sum_{h \in H}\left(S \gamma S\left(g_{i}, h\right)-\overline{S \gamma S}\left(g_{i}\right)\right)^{2}} \sqrt{\sum_{h \in H}\left(S \gamma S\left(g_{i}, h\right)-\overline{S y S}\left(g_{i}\right)\right)^{2}}}
$$

where $\overline{S y S}(g)$ is the average $S y S$ taken over $H$.

Using $\mathrm{SyC}$, we evaluate synteny as an evolutionary signal that can vary across lineages. Note that it is not necessary for $g_{i}$ and $g_{2}$ to be found in synteny; 1 ) similarity to syntenic genes in reference species may support the homology of $g_{i}$ and $g_{2}$ and 2) the range of SyC is 0-1 like NC.

\section{A combined score}

$\mathrm{NC}\left(g_{1}, g_{2}\right)$ and $\operatorname{SyC}\left(g_{1}, g_{2}\right)$ scores are transformed into a single "strength of prediction" score using an elliptical function that evaluates the homology relationship between two genes. This strength of prediction variable has a range between 0 and 1 and increases consistently as $\mathrm{NC}$ and/or SyC values increase. It is standardized, normalized and gives strength of prediction score for all homolog gene pairs. From rigorous testing on a human mouse dataset at different $\mathrm{NC}$ and SyC thresholds (described in Additional File 1), the best curve that has maximum individual family specificity and sensitivity is an ellipse that cuts SyC at around 1.0 and $\mathrm{NC}$ at around 0.5 . For a gene pair $\left(g_{1}, g_{2}\right)$, the formula for calculating the evaluation value $\mathrm{h}\left(g_{1}, g_{2}\right)$ is given by

$$
h\left(g_{1}, g_{2}\right)=N C\left(g_{1}, g_{2}\right)^{2}+0.25 * \operatorname{SyC}\left(g_{1}, g_{2}\right)^{2}-0.25 \text {. }
$$

\section{Gene family clustering}

Depending on the requirement of type of gene families required, we have tested three standard algorithms. GenFamClust has custom implementations of single linkage, complete linkage and average linkage clustering, which are tailored for using transformed scores, are memory efficient and thus suitable for even very large datasets. For single linkage and complete linkage, gene pairs $\left(g_{1}, g_{2}\right)$ with $\mathrm{h}\left(g_{1}, g_{2}\right)>0$ were considered. For average linkage clustering, the average similarity threshold score 0.25 (described in Additional File 1) has been set.

\section{Results}

\section{Validation on a simulated dataset}

To enable validation on data that we fully understand, we generated data using ALF [34], which is a software that simulates major evolutionary forces for genome rearrangement. The details of parameter settings used for generating this dataset are given in Additional File 1.

We selected Mus musculus chromosome 18 as input to ALF due to its nominal size of 497 genes. We then performed six simulations by varying translocation rate, values $0.0002,0.0025$ and 0.005 , and substitution rate, from 100 to 250 PAM, to test GenFamClust for varying levels of gene order and gene content conservation. We used default parameters setting for all other options and turned off parameters related to Gene Inversion, Lateral Gene Transfer (LGT), Fission, Fusion and Pseudogenization events without loss of generality. For this dataset, since no referenced data $\mathrm{R}$ has been defined, Query data $\mathrm{Q}$ also acts as the reference data.

Table 1 illustrates the comparison between NC and GenFamClust for the simulated dataset, where each cell represents the absolute difference in number of true gene families and inferred by using a clustering algorithm on scores from $\mathrm{NC}$ and the combined score ( $\mathrm{NC}$ and SyC). Clearly, GenFamClust outperforms NC in determining the gene families, where the resulting number of gene families formed by GenFamClust is closer to actual gene families in almost all cases. This indicates that $\mathrm{SyC}$ is informative and improves on NC scores alone. Datasets 1, 2 and 3, which have higher synteny conservation, are better approximated by both methods, which emphasizes the dependence of NC and GenFamClust on gene content conservation.

Table 1 Absolute difference between number of gene families determined by NC and those determined by GenFamClust.

\begin{tabular}{cccccccc}
\hline & & \multicolumn{7}{c}{ Dataset } \\
\hline & Transl. rate & .0002 & .0025 & .005 & .0002 & .0025 & .005 \\
\hline & Dupl. rate & .0085 & .0085 &. & .006 & .006 & .006 \\
& Subst. rate & 100 & 100 & 100 & 250 & 250 & 250 \\
\hline Clustering & Algorithm & $\mathbf{1}$ & $\mathbf{2}$ & $\mathbf{3}$ & $\mathbf{4}$ & $\mathbf{5}$ & $\mathbf{6}$ \\
\hline Average Linkage & NC & 83 & 10 & 39 & 1211 & 552 & 493 \\
\hline & GenFamClust & 32 & 7 & 23 & 751 & 457 & 437 \\
\hline Complete & NC & 127 & 35 & 68 & 1316 & 608 & 560 \\
\hline Linkage & & & & & & & \\
\hline Single Linkage & NenFamClust & 58 & 16 & 53 & 821 & 503 & 489 \\
\hline & GenFamClust & 6 & 16 & 1 & 631 & 397 & 379 \\
\hline Extant gene \\
families
\end{tabular}

Each cell represents the absolute difference between number of extant gene families (observable at leaves) (last row) and the number of gene families determined by the corresponding gene family algorithm for the corresponding dataset with the corresponding linkage algorithm. For NC, the threshold of 0.5 was used while for GenFamClust, the elliptical curve with NC $=0.5$ and $\mathrm{SyC}=1.0$ was used. 


\section{Human versus mouse dataset}

The Human-Mouse dataset is from Ensembl Genes 69 [35], has human and mouse genomes as query, and has a reference dataset consisting of complete genomes from eighteen eukaryotic species, ranging from yeast to mammals(including human and mouse). A gold standard dataset was available in the form of twenty homologous gene families of human and mouse identified by Song et al. [16].

Since GenFamClust requires whole genome information, we used the human and mouse genome data, extracted from Ensembl, as our query sequences. For reference sequences, we selected genomes evenly distributed over the Species tree of life provided by Ensembl [36].

Song et al. suggested 20 gene families in human and mouse based on literature in their paper [16]. These families are diverse and contain single as well as multidomain families; contain very small families to very large families; and vary from very conserved families to highly divergent sequence families (shown in Additional File 1). With this known excellent gold standard, it was very logical to test our approach on this dataset and compare with similarity only software.

\section{Validating GenFamClust}

GenFamClust was applied to the human and mouse dataset and was checked for the results on the gold standard data of twenty families. The first paper published after sequencing of mouse genome gave a synteny-based match of mouse genome with the human genome [37]. Such a large number of conserved syntenic regions and the level of conservation provides a strong argument in favor of using synteny to support gene homology inference. To validate that the synteny score of GenFamClust is capturing gene order conservation information, we applied GenFamClust on the human and mouse datasets and found that GenFamClust could replicate the original image [37] almost perfectly: 342 syntenic segments with 217 blocks of consistent color in the original image vs 294 syntenic segments with 208 blocks of consistent color using $\mathrm{NC}$ and SyC). The few regions and segments missed by our approach did not contain genes or contained less than five genes. Figure 2 is a comparison between the original image and our results.

\section{Comparison with Neighborhood Correlation without synteny}

We applied GenFamClust and NC to complex and diverse cases of the gold standard dataset from Song et. al [16]. We compared the performance of Neighborhood Correlation software to the performance of GenFamClust according to $F(i, j)$, the harmonic mean of precision $(P(i$, $j)=$ fraction of elements in cluster $j$ that are members of family $i)$ and recall $(R(i, j)=$ fraction of members of family $i$ that are found in cluster $j$ ) [31]. $F(i, j)$ (shown in Figure 3 ) is determined by following formula.

$$
f(i, j)=\frac{2 P(i, j) R(i, j)}{P(i, j)+R(i, j)}
$$

The results, shown in Figure 3, clearly demonstrate that we have a marked improvement in terms of accuracy for single linkage (on average 3.81 percentage points) and average linkage clustering (on average 1.63 percentage points) while we have maintained the accuracy shown by the Neighborhood Correlation alone in the complete linkage-clustering (on average 0.44 percentage points) algorithm. In particular comparing the two quality scores at the proposed threshold of 0.5 for NC, GenFamClust outperforms NC in single linkage (2.37 percentage points) and average linkage ( 0.23 percentage points) clustering while has a minute difference $(-0.1$ percentage points) with $\mathrm{NC}$ in complete linkage clustering.

We also examined the effect of varying $\mathrm{NC}$ values while SyC score remains constant at 1.0 and vice versa. Lowering $\mathrm{NC}$ threshold improves the overall and all-kinase quality scores. However, it can be observed that the small sized families tend to suffer with low NC values. Therefore, it is logical to choose a NC value threshold that is best able to define individual families for all three clustering algorithms. In this regard, a NC value threshold of just around 0.5 seems to be the most appropriate (complete tables in Additional File 1). Joseph et al. made the same deduction in the follow up paper of Neighborhood Correlation as well [31]. Similarly, for GenFamClust and $\mathrm{NC}$ value 0.5 , an evaluation curve cutting SyC axis at 1.0 on $\mathrm{SyC}$ seems to provide the best results (Data and tables in Additional File 1).

\section{Discussion}

Conserved gene order is one of the properties that can aid in identifying homologs along with similarity. In this paper, we combined gene order and content conservation to infer homology. We use the concept of local synteny as well as gain evidence from multiple genomes, similar to $[29,30]$. However, we suggest a way to quantify synteny and combine it with similarity information before doing the actual classification. Moreover, we avoid the pitfalls of BLAST scores by building on NC [31].

\section{Syntenic orthologs versus non-syntenic Orthologs}

Since orthology is generally extracted from direct similarity measures, orthologs with syntenic support have an extra degree of confidence in their prediction. Depending on the requirements for determining gene families, if split families is not problematic but accurate clustering is a requirement, then syntenic orthologs can act as a good dataset. Furthermore, as displayed by Wolf et al. [38], syntenic orthologs can act as validation data for confirming the results from different techniques. 

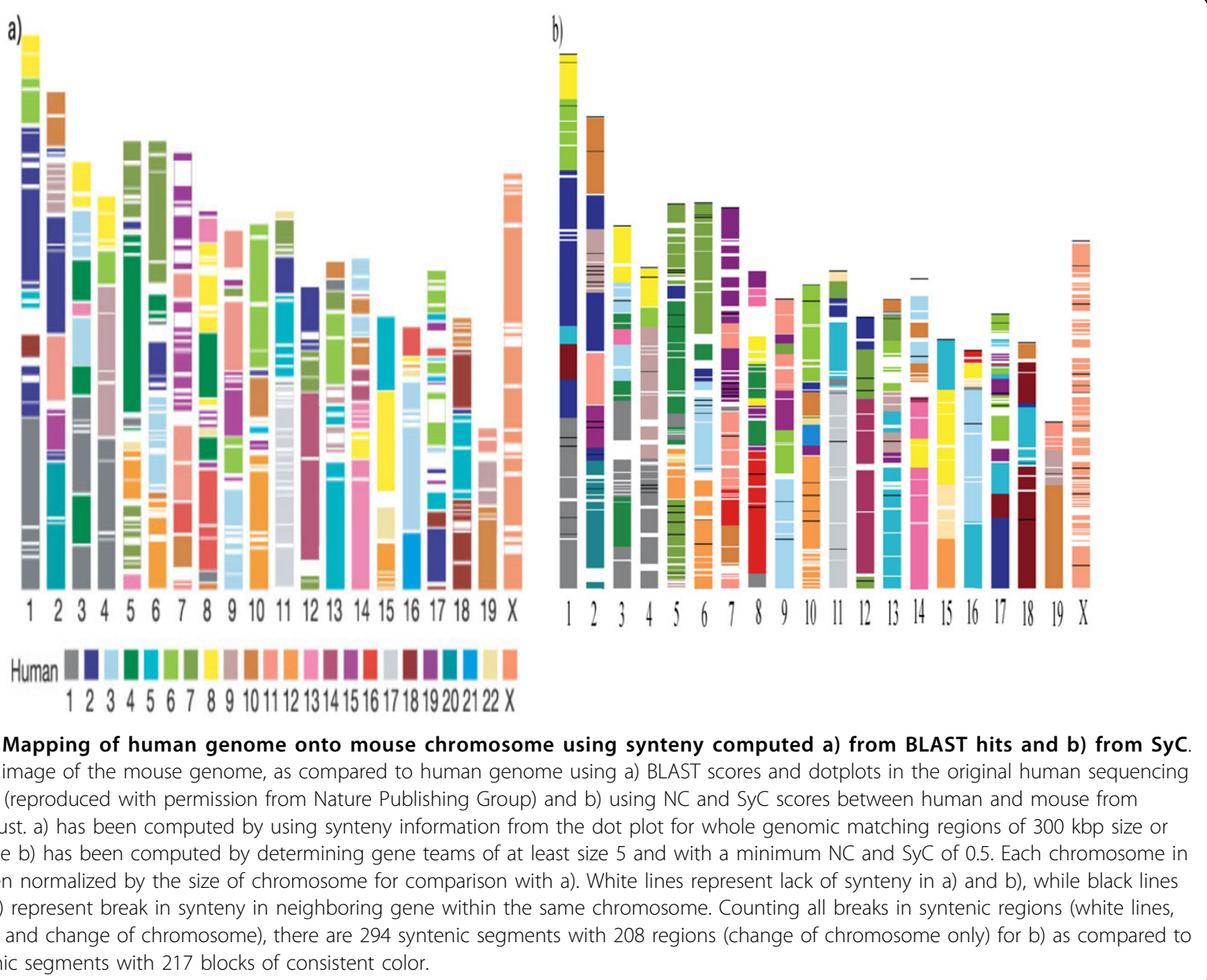

\section{Choice of reference dataset}

The choice of reference dataset is highly important as it has profound impact on the Neighborhood Correlation scores for both similarity and synteny. The reference data must reflect the similarity and synteny information for the query dataset accurately. While there is no upper bound on the amount of reference data, there are practical limitations as well as usability issues for the size of the reference data set; having many species with little divergence times will have redundant similarity and synteny information, which only adds to the computational burden without adding any new information. On the other side of spectrum, if no reference data is available, the query data itself serves as reference data. In general, reference data should be able to capture the synteny and similarity relationships for the query data e.g. by choosing a few representative species from each branch of a known species tree from which query dataset is taken from.

\section{Advantages of using Query versus Reference Blast}

All similarity-based programs mentioned in this study require All-versus-All Blast results for gene family classification. GenFamClust takes advantage of network structure employed in NC for similarity and performs a Query versus Reference Blast only. Then, the Reference versus Reference Blast results are appended to these results and passed onto the next module for NC calculation. As the size of $R$ is fixed, the size of $Q$ varies and is the determining factor of the time taken by the Blast module. While for an All-versus-All Blast, it would take $O\left((n+m)^{2}\right)$ time, this version of Blast takes $O\left(m n+n^{2}\right)$ time, where $m$ is the size of $Q$ and $n$ is the size of $R$. This comparison is, of course, only meaningful when $m>>n$. Furthermore, the Blast results for Reference versus Reference dataset can be reused giving the effective time complexity of $O(\mathrm{~nm})$.

\section{Conclusions}

Clustering sequences into meaningful families and to infer the true evolutionary history of widely diverse set of genes are difficult tasks. While the clustering techniques are relatively long known and mostly standard, homology inference is the defining step for determining accurate gene families. However, homology inference is 
a)

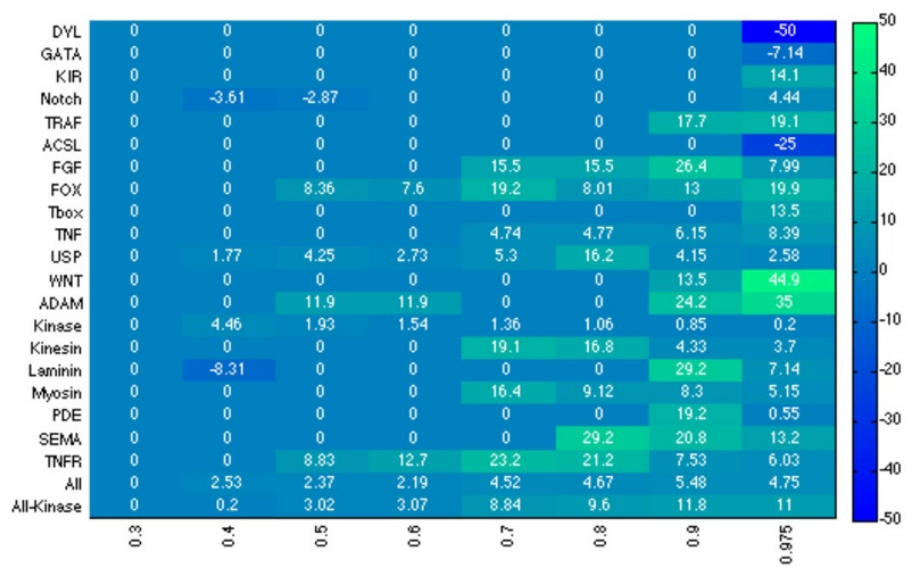

b)

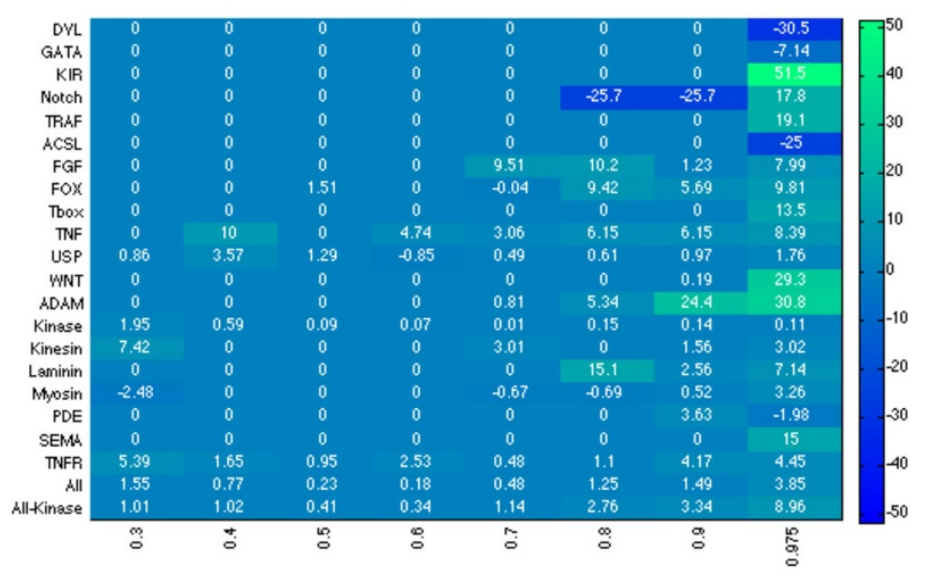

C)

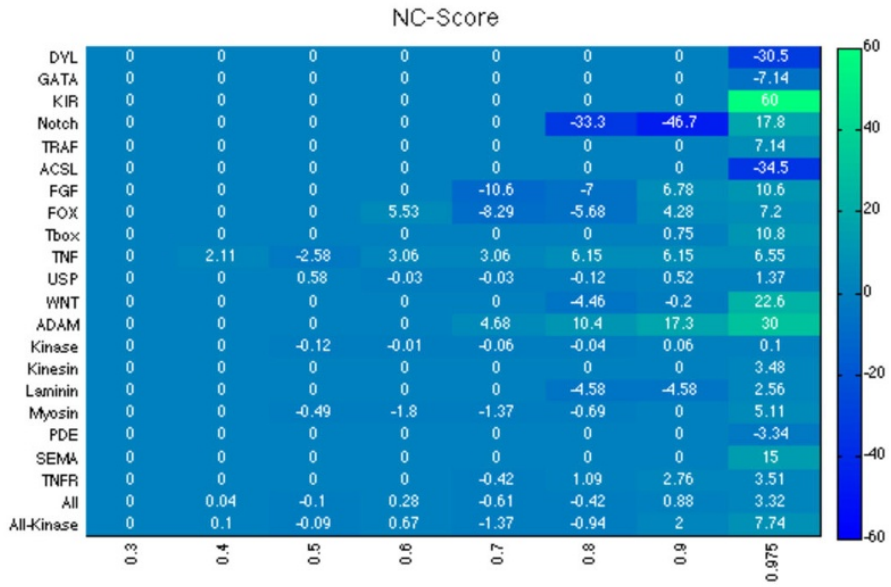

NC-Score

Figure 3 Evaluation of clustering on transformed scores at various NC scores with SyC cut at 1.0 versus NC scores alone. Figure enumerates and displays the comparison of gene families formed by a) Single Linkage Clustering, b) Average Linkage and c) Complete Linkage. The value in each cell represents the difference between quality scores of clusters generated by GenFamClust and quality scores of clusters generated by NC alone for corresponding cell on human mouse test dataset. Green cells represent the families where GenFamClust outperforms the NC method, dark blue cells represent the families where NC outscores GenFamClust, and blue cells represent the families where both quality scores are equal. The intensity of green and blue indicates the difference in percentage between the two approaches, where darker color shows greater difference. 
an Achilles heel of determining reliable gene families. Methodologies only based on similarity have long been proposed for homology inference without taking account of synteny. However, a sensible combination of sequence similarity and synteny would perform better than only similarity-based approaches. In this work, we have proposed GenFamClust, a novel pipeline that is first to make use of network structure of synteny across multiple genomes. It provides an objective way of assessing synteny for a gene pair as well as a noticeable improvement in accuracy as compared to a similarityonly algorithm. We suggest that GenFamClust is a good framework due to its ability to handle larger genomes, large and diverse datasets spread across a variety of species from Eukaryotes, as well as across varying protein domain architectures from single domain to conserved and varying multi-domain proteins. Another feature of GenFamClust is its ability to work and define synteny with fragmented gene assemblies. Moreover, the Java implementation of GenFamClust is user friendly and easy to deploy and use by the general community.

\section{Additional material}

Additional file 1: Supplementary materials. Data descriptions, technical details, and additional results.

\section{Competing interests}

The authors declare that they have no competing interests.

\section{Authors' contributions}

RHA designed and implemented the algorithm for GenFamClust, prepared the multi-species datasets, performed comparative analysis with NC and drafted the manuscript. SAM collaborated in designing the algorithm, performed statistical analysis of all datasets and prepared the simulated dataset. MAK participated in the design of the study and aided in evaluation of human-mouse dataset. LA conceived of the study, participated in its design and coordination and helped to draft its manuscript.

\section{Acknowledgements}

We thank Pekka Parviainen and Kristoffer Sahlin for help and suggestions, and Daniel Dalquen for help with running ALF. The computations were performed on resources provided by SNIC through Uppsala Multidisciplinary Center for Advanced Computational Science (UPPMAX) in project b2012160. Pontus Freyhult and Lennart Karlsson at UPPMAX are acknowledged for assistance concerning technical aspects in making the code run on the UPPMAX resources.

\section{Declarations}

RHA was funded by Higher Education Commission of Pakistan (HEC). SAM was funded by EuroSPIN (an Erasmus Mundus joint doctoral program) and Swedish e-Science Research Center (SeRC). MAK was funded by UET Peshawar. Publication charges for this article were paid by the KTH Royal Institute of Technology.

This article has been published as part of BMC Bioinformatics Volume 14 Supplement 15, 2013: Proceedings from the Eleventh Annual Research in Computational Molecular Biology (RECOMB) Satellite Workshop on Comparative Genomics. The full contents of the supplement are available online at http://www.biomedcentral.com/bmcbioinformatics/supplements/ $14 / 515$.

\section{Authors' details}

${ }^{1} \mathrm{KTH}$ Royal Institute of Technology, School of Computer Science and Communication, Department of Computational Biology, Stockholm, Sweden. ${ }^{2}$ Science for Life Laboratory, Karolinska Institutet Science Park, Solna, Sweden. ${ }^{3}$ Swedish e-Science Research Center, Sweden. ${ }^{4}$ Department of Numerical Analysis and Computer Science, Stockholm University, SE-100 44, Stockholm, Sweden.

Published: 15 October 2013

References

1. Fitch WM: Distinguishing homologous from analogous proteins. Systematic Zoology 1970, 19(2):99-113

2. Camacho C, Coulouris G, Avagyan V, et al: BLAST+: architecture and applications. BMC Bioinformatics 2009, 10:421.

3. Altschul SF, Madden TL, Schäffer AA, Zhang J, Zhang Z, Miller W, and Lipman DJ: Gapped BLAST and PSI-BLAST: a new generation of protein database search programs. Nucleic Acids Res. 1997, 25(17):3389-3402.

4. Overbeek $\mathrm{R}$, Fonstein $\mathrm{M}$, et al: The use of gene clusters to infer functional coupling. Proc Natl Acad Sci 1999, 96:2896-2901.

5. Tatusov RL, Koonin EV, and Lipman DJ: A genomic perspective on protein families. Science 1997, 278:631-637.

6. Miele $V$, Penel $S$, and Duret $L$ : Ultra-fast sequence clustering from similarity networks with SiLiX. BMC Bioinformatics 2011, 12:116.

7. BLASTCLUST. [http://www.ncbi.nlm.nih.gov/BLAST/].

8. Sibson R: SLINK: an optimally efficient algorithm for the single-link cluster method. The Computer Journal (British Computer Society) 1973, 16(1):30-34.

9. Kristensen DM, Wolf $\mathrm{YI}$, et al: Computational methods for Gene Orthology inference. Briefing in Bioinformatics 2011, 12(5):379-91.

10. Wolf Yl, Novichkov PS, Karev GP, et al: The universal distribution of evolutionary rates of genes and distinct characteristics of eukaryotic genes of different apparent ages. PNAS 2009, 106(18):7273-80.

11. Koonin EV, and Wolf Yl: Genomics of bacteria and archaea: the emerging dynamic view of the prokaryotic world. Nucleic Acids Res 2008, 36(21):6688-719.

12. Enright AJ, Dongen VS, and Ouzounis CA: An efficient algorithm for largescale detection of protein families. Nucleic Acids Res 2002, 30(7):1575-84.

13. Li L, Stoeckert CJ, and Roos DS: OrthoMCL: identification of ortholog groups for eukaryotic genomes. Genome Research 2003, 13(9):2178-89.

14. Remm M, Storm CEV and Sonnhammer ELL: Automatic clustering of orthologs and in-paralogs from pairwise species comparisons. Journal of Molecular Biology 2001, 314(5):1041-1052.

15. Alexeyenko A, Tamas I, et al: Automatic clustering of orthologs and inparalogs shared by multiple proteomes. Bioinformatics 2006, 22:e9-e15.

16. Song $N$, Joseph JM, Davis $G B$, and Durand D: Sequence similarity network reveals common ancestry of multidomain proteins. PLOS Computational Biology 2008, 4(4):e1000063.

17. Miele V, Penel S, Daubin V, Picard F, Kahn D, and Duret L: High-quality sequence clustering guided by network topology and multiple alignment likelihood. Bioinformatics 2012, 28(8):1078-85.

18. Bhardwaj $G$, Ko KD, et al: PHYRN: a robust method for phylogenetic analysis of highly divergent sequences. PloS ONE 2012, 7(4):e34261.

19. Jothi R, Zotenko E, Tasneem A, and Przytycka TM: COCO-CL: hierarchical clustering of homology relations based on evolutionary correlations. Bioinformatics 2006, 22(7):779-88.

20. Friedman R, and Hughes AL: Gene duplication and the structure of eukaryotic genomes. Genome Res 2001, 11:373-81.

21. Heber S, and Stoye J: Algorithms for finding gene clusters. WABI Volume 2149 of Lecture Notes in Computer Science 2001, 254-265.

22. Luc N, Risler J, et al: Gene teams: a new formalization of gene clusters for comparative genomics. Comput Biol Chem 2003, 27:59-67.

23. Lehmann J, Stadler PF, and Prohaska SJ: SynBlast: Assisting the analysis of conserved synteny information. BMC Bioinformatics 2008, 9:351.

24. Wang $Y$, Tang $H$, et al: MCScanX: a toolkit for detection and evolutionary analysis of gene synteny and collinearity. Nucleic Acids Res 2012, 40(7):e49.

25. Rödelsperger C, Dieterich C: CYNTENATOR: progressive gene order alignment of 17 vertebrate genomes. PLOS ONE 2010, 5(1):e8861.

26. Haas BJ, Delcher AL, et al: DAGchainer: a tool for mining segmental genome duplications and synteny. Bioinformatics 2004, 20(18):3643-3646. 
27. Wapinski I, Pfeffer A, Friedman N, and Regev A: Automatic genome-wide reconstruction of phylogenetic gene trees. Bioinformatics 2007, 23(13): i549-58.

28. Åkerborg Ö, Sennblad B, Arvestad L, and Lagergren J: Simultaneous Bayesian gene tree reconstruction and reconciliation analysis. PNAS 2009, 106(14):5714-5719.

29. Jun J, Mandoiu II, and Nelson CE: Identification of mammalian orthologs using local synteny. BMC Genomics 2009, 10:630.

30. Sarkar A, Soueidan $H$, and Nikolski M: Identification of conserved gene clusters in multiple genomes based on synteny and homology. BMC Bioinformatics 2011, 12(Suppl 9):S18.

31. Joseph $J M$, and Durand $D$ : Family classification without domain chaining. Bioinformatics 2009, 25(12):i45-53.

32. Sorensen T: A method of establishing groups of equal amplitude in plant sociology based on similarity of species and its application to analyses of the vegetation on Danish commons. Biologiske Skrifter 1948, 5:1-34.

33. Sokal $R$, and Michener C: A statistical method for evaluating systematic relationships. University of Kansas Science Bulletin 1958, 38:1409-1438.

34. Dalquen DA, Anisimova M, Gonnet GH, Dessimoz C: ALF - A Simulation Framework for Genome Evolution. Mol Biol Evol 2012, 29(4):1115-1123.

35. Flicek P, Amode MR, Barrell D, et al: Ensembl 2012. Nucleic Acids Research 2012, 40(Database):D84-D90.

36. Species tree of species present in Ensembl as generated by Ensembl Compara. [http://www.ensembl.org/info/about/species_tree.pdf].

37. Waterston RH, Lindblad-Toh $\mathrm{K}$, et al: Initial sequencing and comparative analysis of the mouse genome. Nature 2002, 420(6915):520-62.

38. Wolf $\mathrm{Yl}$, and Koonin $\mathrm{EV}$ : A tight link between orthologs and bidirectional best hits in bacterial and archaeal genomes. Genome Biol Evol. 2012, 4(12):1286-94.

doi:10.1186/1471-2105-14-S15-S12

Cite this article as: Ali et al: Ouantitative synteny scoring improves homology inference and partitioning of gene families. BMC Bioinformatics 2013 14(Suppl 15):S12.

\section{Submit your next manuscript to BioMed Central and take full advantage of:}

- Convenient online submission

- Thorough peer review

- No space constraints or color figure charges

- Immediate publication on acceptance

- Inclusion in PubMed, CAS, Scopus and Google Scholar

- Research which is freely available for redistribution

Submit your manuscript at www.biomedcentral.com/submit 